\title{
Duplication of the inferior vena cava: Case report and review of the literature
}

\author{
Meriam Sabbah ${ }^{1}$, Khaoula Chabbouh ${ }^{2}$, Nawel Bellil ${ }^{1}$, Norsaf Bibani ${ }^{2}$, fatma dieb ${ }^{2}$, maroua \\ ben ali $^{2}$, Dhafer Haddad ${ }^{2}$, and Dalila Gargouri² \\ ${ }^{1}$ Hopital Habib Thameur \\ ${ }^{2}$ Habib Thameur Hospital
}

September 11, 2020

\begin{abstract}
A 33-year-old man was admitted to the hospital for bowel obstruction secondary to fistulizing and stenosing Crohn's disease. A computerized tomography scan of abdomen showed a duplication of the inferior vena cava.
\end{abstract}

\section{Introduction :}

The inferior vena cava duplication may occur in $0,7 \%$ of general population (1), often fortuitously discovered during radiological exams (2). It can be mistaken for retroperitoneal adenopathy. It is important to be aware about this abnormality in order to avoid hemorrhagic complications during retroperitoneal surgery. A case of a patient with Crohn's disease who was candidate for surgical treatment in whom an inferior vena cava duplication was incidentally discovered on the CT scan is reported.

\section{Case report:}

A 33-year-old man presented to the emergency department with an acute mechanical bowel obstruction. He reports also burning micturition. The patient had a past medical history of fistulizing and stenosing Crohn's disease with anoperineal manifestations which was untreated. He has not consulted for 5 years. He smokes 10 pack-year and consumes cannabis occasionally. On examination, there was no fever. Vital signs were stable. The abdomen was distended and tympanic. The anal examination shows 7 productive fistulous orifices. The white blood cells count was 10100 per microliter. The hemoglobin level was $12 \mathrm{~g} / \mathrm{dL}$ and the C-reactive protein was $78 \mathrm{mg} / \mathrm{L}$. An abdomen radiography without preparation was performed showing gas-fluid levels. A computerized tomography scan of abdomen with IV contrast showed a CT appearance of chronic inflammatory bowel complicated by ileo-ileal, ileo-vesical and multiple perineal fistulas without intra-abdominal or parietal collection. It was also noted a duplication of the inferior vena cava with a left vena cava drained in the homolateral renal vein (Figure 1 and 2). The patient was scheduled for surgery (ileocoecal resection, disconnection of the ileovesical fistula and placement of setons). Post operative outcome was favorable especially no hemorrhagic complications occured. An anti-TNF therapy after surgery was also scheduled due to perineal fistula.

\section{Discussion:}

The inferior vena cava (IVC) allows the drainage of venous blood from the lower limbs and abdominal organs to the right atrium. Knowledge of its anatomical variants is essential for subsequent therapeutic management (3). So that hemorrhagic complications during retroperitoneal surgery can be avoided. The IVC variations are frequently detected by CT scan as it is widely used to explore diverse abdominal symptoms. The IVC is formed essentially of 4 segments: the hepatic, suprarenal, renal, and infrarenal IVC (4). During embryonic 
life, the IVC genesis occurs between weeks 6 and 10 of gestation. It is a complex procedure involving different anastomosis and veins regression. The persistence of both supracardinal veins forming duplicated infrarenal IVC segments leads to IVC duplication (5). The left infrarenal IVC joins the left renal vein and drains into a normal suprarenal IVC. This abnormality is found in $0.2-3 \%$ of the general population (5). It is classified in 3 types. The major duplication or type I, where 2 symmetric trunks are observed with the same caliber as the preaortic trunk. The minor duplication or type II, where also two symmetric trunks but which are smaller than the preaortic trunk. And the asymmetric duplication or type III, with a smaller left IVC and a larger right (6).

The diagnosis of IVC duplication happens usually during retroperitoneal surgery as it is often asymptomatic. But it can have clinical impact such as recurrent thromboembolism despite an IVC filter (7). In other instances like transplant surgery, it is important de be aware of this anatomical variantion (7). It is also important to be aware about this abnormality in order to avoid hemorrhagic complications during retroperitoneal surgery (9). Fortunately, in our case, the post operative outcome was favorable and no bleeding was noted.

\section{Conclusion:}

Although the literature search dealing with the complications of the IVC duplication was poor (8), surgeons involved in retroperitoneal procedures should be awared of different vena cava anatomical variations in order to minimize morbidity such as post operative bleeding.

\section{References:}

1. Hostiuc S, Minoiu C, Negoi I, Rusu MC, Hostiuc M. Duplication and transposition of inferior vena cava: A meta-analysis of prevalence. J Vasc Surg Venous Lymphat Disord. 2019;7(5):742-55.

2. Ueda J, Hara K, Kobayashi Y, Ohue S, Uchida H. Anomaly of the inferior vena cava observed by CT. Comput Radiol. 1983;7(3):145-54.

3. Smillie RP, Shetty M, Boyer AC, Madrazo B, Jafri SZ. Imaging evaluation of the inferior vena cava. Radiographics. 2015;35(2):578-92.

4. Bass JE, Redwine MD, Kramer LA, Huynh PT, Harris JH, Jr. Spectrum of congenital anomalies of the inferior vena cava: cross-sectional imaging findings. Radiographics. 2000;20(3):639-52.

5. Phillips E. Embryology, normal anatomy, and anomalies. In: Ferris EJ, Hipona FA, Kahn PC, Phillips E, Shapiro JH, eds. Venography of the inferior vena cava and its branches. Baltimore, Md: Williams \& Wilkins, 1969; 1-32.

6. Shaha P, Garg A, Sahoo K, Kothari N, Garg P. Duplication of inferior vena cava with associated anomalies: a rare case report. J Clin Diagn Res 2016;10:TD01-4.

7. Chiarugi M, Fregoli L, Iacconi P. Inferior vena cava duplication. Updates Surg. 2015;67(3):325-7.

8. Cossu ML, Ruggiu M, Fais E, sparta C, Dettori G, Noya G. Le anomalie congenite della vena cava inferior. Minerva Chir 2000; 55: 703-708.

9. Christakis PG, Cimsit B, Kulkarni S (2012) Complication arising from a duplicated inferiro vena cava following laparoscopic living donor nephrectomy: a case report. Transplant Proc 44:1450-14

Figure 1: CT scan (sagittal plane): Duplication of the inferior vena cava 


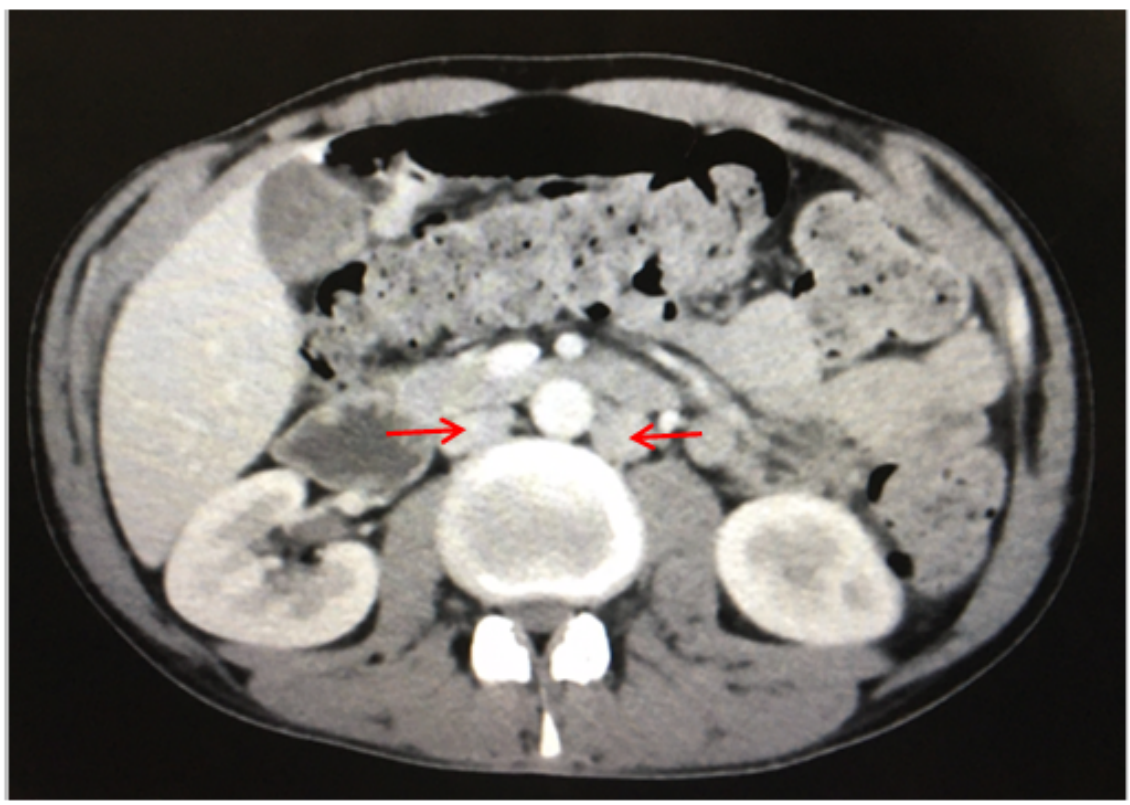

Figure 2: CT scan (coronal plane): Duplication of the inferior vena cava 


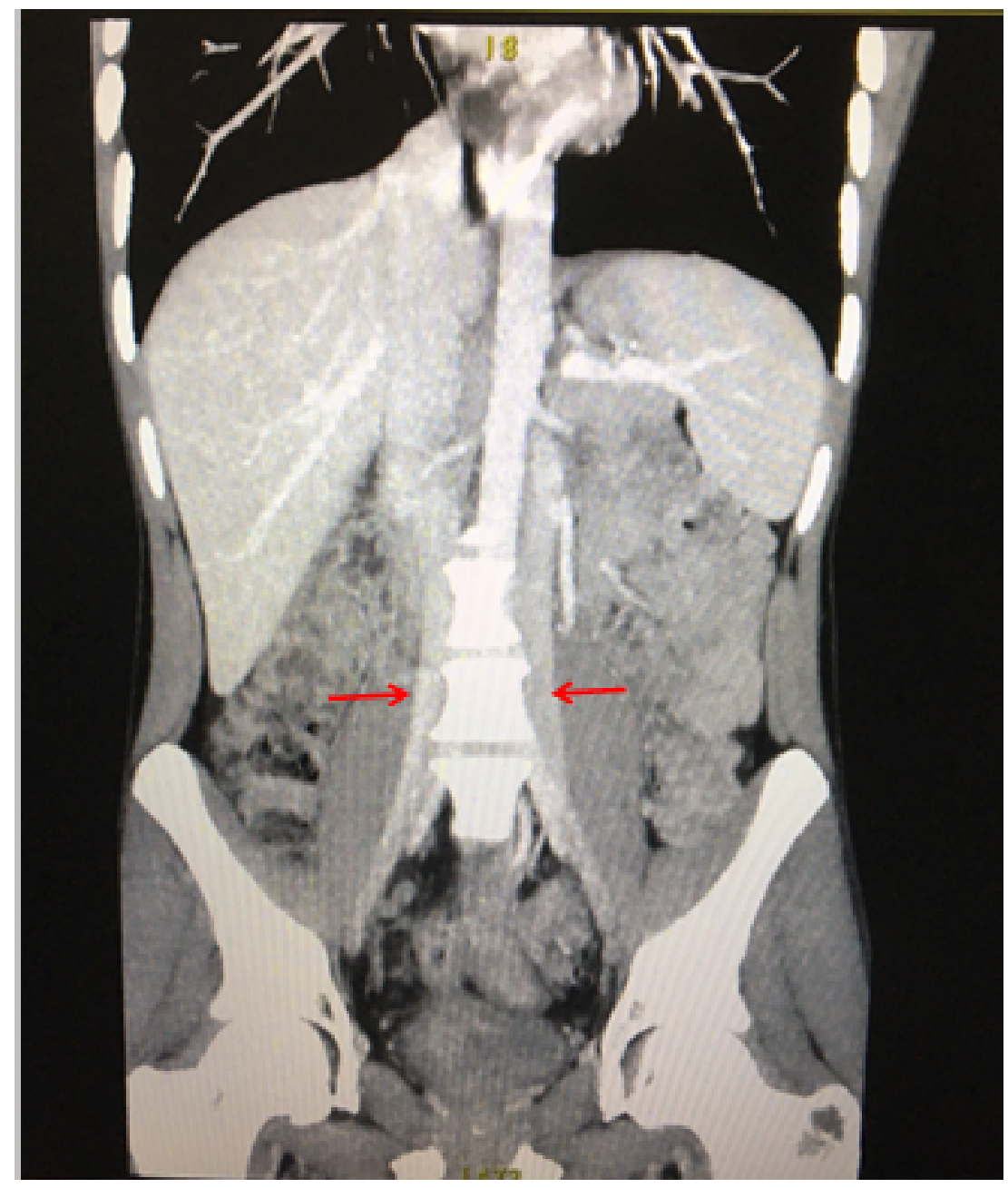




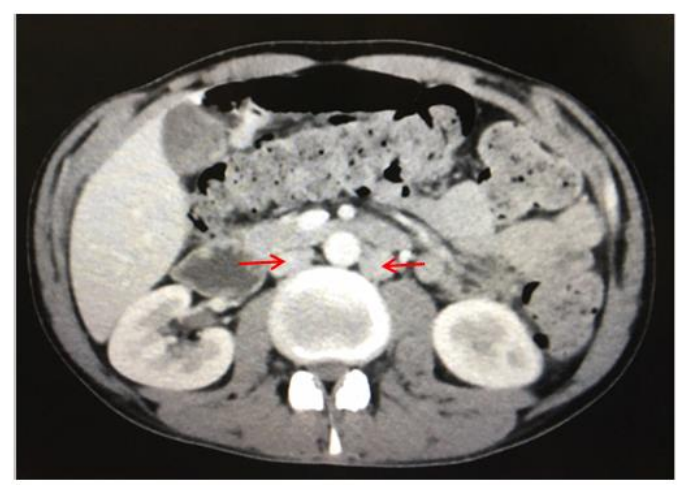

Figure 1: CT scan (sagittal plane): Duplication of the inferior vena cava

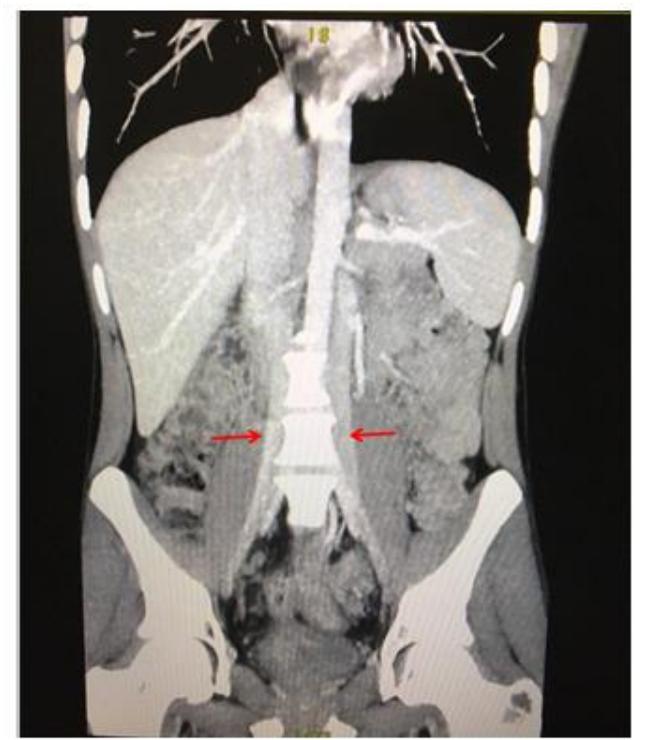

Figure 2: CT scan (coronal plane): Duplication of the inferior vena cava 